\title{
Hacia un nuevo paradigma de aprendizaje de segundas lenguas móvil, abierto y social
}

\section{Towards a New Learning Paradigm for Mobile, Open and Social Second Languages}

\author{
Elena Bárcena*
}

Universidad Nacional de Educación a Distancia, España

ORCID: https://orcid.org/0000-0002-0645-5933

Recibido 05-11-19 Revisado 25-11-19 Aprobado 11-01-20 En línea 26-02-20

*Correspondencia

Email: mbarcena@flog.uned.es
Citar como:

Bárcena, E (2020). Hacia un nuevo paradigma de aprendizaje de segundas lenguas móvil, abierto y social. Propósitos y Representaciones, 8(1), e460. doi: http://dx.doi.org/10.20511/pyr2020.v8n1.460 


\section{Resumen}

Este artículo explora el diseño y desarrollo de un innovador paradigma didáctico para segundas lenguas a distancia, social y abierto (con una triple dimensión lingüística, metodológica y tecnológica), cuyo núcleo quede definido por la tecnología móvil y el Aprendizaje de Lenguas Asistido por Móviles o MALL, no sólo como una herramienta adicional que puede ser utilizada para desempeñar ciertas tareas (de manera más o menos estructurada), sino como la principal forma en la que los estudiantes a distancia de segundas lenguas pueden interaccionar y llevar a cabo su aprendizaje de manera efectiva.

Palabras Clave: Aprendizaje; Aprendizaje de lenguas; Tecnología móvil; Educación a distancia.

\section{Summary}

This article explores the design and development of an innovative didactic paradigm for second languages at a distance, social and open (with a triple linguistic, methodological and technological dimension), whose core is defined by mobile technology and the Learning of Languages Assisted by Mobile or MALL, not only as an additional tool that can be used to perform certain tasks (in a more or less structured way), but as the main way in which distance students of second languages can interact and carry out their learning in a way effective.

Keywords: Learning; Language Learning; Mobile Technology; Long Distance Education.

\section{Introducción}

En materia de aprendizaje de segundas lenguas, en primer lugar, partimos de la base, comúnmente aceptada por la comunidad experta, de que no sólo se basa en el conocimiento, en el sentido de que requiere la asimilación pasiva de vocabulario y de reglas combinatorias, sino que se basa principalmente en el empleo de habilidades, puesto que implica poner en práctica una compleja matriz de capacidades funcionales receptivas, productivas e interactivas verbales (y no verbales), cuyo papel en el éxito general del acto comunicativo se considera generalmente más prominente que el de los elementos formales u organizativos ${ }^{1}$. Relacionado con el punto anterior, asumiendo que el objetivo de las lenguas es su uso en la comunicación humana, es razonable inferir que su aprendizaje debería implicar una práctica considerable de dicha comunicación, al igual que un estudiante debe tocar el piano para convertirse en pianista o sacar fotografías para convertirse en fotógrafo. Por lo tanto, puesto que la práctica comunicativa verbal es intrínsecamente social, una parte importante de ella debe ser emprendida con otros interlocutores.

Además, si se espera que el estudiante con éxito de una segunda lengua asuma un rol activo en su propio aprendizaje, al ser el conocimiento generalmente construido por el sujeto en lugar de transferido a él, requiere la oportunidad de construir estrategias y conexiones que sean significativas en su caso y en ese preciso momento (Cuesta Medina, Anderson \& McDougald, 2017). Sin embargo, dada la naturaleza intrínsecamente social de la comunicación verbal, la comunidad investigadora valora procesos como la negociación de significado y la participación en un trabajo de grupo estructurado, a veces orientado al desempeño de tareas, orientado al cumplimiento de objetivos, participando, prestándose asistencia mutua y construyendo y compartiendo nuevos conocimientos y habilidades en colaboración con otros (Warschauer y Kern, 2000).

Sin embargo, las lenguas tienen una doble naturaleza como vehículos comunicativos verbales y también sistemas gobernados por reglas. Después de la infancia, los expertos coinciden

\footnotetext{
${ }^{1}$ La investigación conducente a este artículo ha sido realizada en el contexto del proyecto SWITCHEDON (( $n^{\circ}$ ref.: FFI2016-80613-P), gracias a una subvención del Ministerio de Ciencia, Innovación y Universidades de España.
} 
en que los individuos pierden gradualmente parte de las habilidades innatas de adquisición de lenguas y adquieren un perfil cognitivo basado en reglas. Por lo tanto, es probable que el estudiante adulto de una segunda lengua se beneficie del modelo explícito de aprendizaje, algo parcialmente basado en explicaciones presenciales/textuales/visuales/auditivas con ejemplos ilustrativos seguidos de alguna forma creativa y atractiva de práctica (Ellis, 2015). Se ha argumentado que parte de este proceso será más eficaz si se realiza individualmente (Bárcena, Read \& Cuesta Medina, 2019), en particular para mejorar ciertas áreas lingüísticas, como la pronunciación o la puntuación, ya que proporciona la flexibilidad necesaria y la adaptación a los estilos de aprendizaje personal, preferencias, ritmos y circunstancias, a la vez que mejora el desarrollo de habilidades y procesos metacognitivos y autorreguladores (Read y Barcena, 2014). No hay dos personas que tengan exactamente el mismo perfil de aprendizaje (en términos de metas, habilidades, intereses, etc.) y esta diversidad debe ser respetada y atendida siempre que sea posible.

El aprendizaje a distancia se ha debatido en la literatura especializada como una modalidad en la que los estudiantes pueden alcanzar con eficacia una enorme diversidad de objetivos formativos (García Aretio, 2017). Es rentable en muchos casos en términos de flexibilidad, conveniencia y ahorro de tiempo. Lo que es más importante, tiene un gran potencial específicamente para las segundas lenguas, ya que ofrece una oportunidad para integrar los prometedores avances que están surgiendo en la web (redes sociales, gamificación, etc.) con las plataformas educativas en línea y las prácticas metodológicas que va estableciendo el profesor. De hecho, una propuesta docente innovadora y motivadora de la tecnología en el estudio a distancia es casi "esperado" en estos días por parte de todas las demás partes involucradas, incluyendo los estudiantes y las autoridades educativas.

Muchos autores, como Hurd, Beaven y Ortega (2001), publicaron hace tiempo profusamente acerca de la viabilidad de la enseñanza de segundas lenguas a distancia, y en particular del inglés, cuando la asistencia continuada a clases presenciales no es viable. Desde entonces, estos y otros investigadores (Moore \& Kearsley, 2011) han centrado sus esfuerzos en recabar evidencia de esto y en refinar los métodos, prácticas y estrategias para la enseñanza a distancia de segundas lenguas con enfoques multidisciplinares y diseños y herramientas cada vez más sofisticados, de tal forma que la enseñanza de una segunda lengua hoy es más una opción libre que un "sucedáneo formativo". Por todo ello, hoy puede afirmarse con rotundidad que la enseñanza de una segunda lengua a distancia no es necesariamente un complemento ni un suplemento de la enseñanza presencial tradicional en el aula, sino que tiene un estatus paralelo a esta. La incorporación de la tecnología en línea ha posibilitado un aprendizaje a distancia práctico y abstracto, exploratorio y mecánico, interactivo y colaborativo, y también adaptativo (en ritmo y nivel) y personalizado (según intereses y objetivos). A través de la interacción mediada por la tecnología, se posibilita, además, el desarrollo de las llamadas soft skills o habilidades sociales, que permiten a su vez el crecimiento personal y profesional, en áreas como la empleabilidad, el desarrollo social, la participación, el cambio y la adaptación (García, López, Molina \& Morales, 2016). Naturalmente, no se trata de una modalidad didáctica desprovista de retos. Durán Padilla y Reséndiz Rodríguez (2007) mencionan que, dado que el estudiante no cuenta con la proximidad emocional de profesor y compañeros, el fracaso académico y el abandono pueden llegar si aquel cae en la pasividad y la apatía. Sin embargo, el problema más común suele estar relacionado con los fallos tecnológicos, el deficiente o rudimentario diseño de programas o al menos su falta de robustez e inadecuado funcionamiento del software (Bates, 2005).

En cuanto a los denominados nuevos roles del profesor de segundas lenguas a distancia y en línea, Pérez, Bernabeu, González, Gión, Navarro y Heliz (2012) comentan que "En [la] construcción personal [del saber], el papel del profesor consiste en guiar, orientar, acompañar, sostener y potenciar los esfuerzos de aprendizaje que el estudiante realiza". De los muchos autores que han estudiado dichos roles, una de los que lo ha hecho de una forma más sintética y elocuente ha sido King (1993) al señalar que el profesor a distancia debía ser, no un sabio en el escenario, sino un guía al lado" (en inglés "not a sage on the stage, but a guide on the side"). Sin embargo, cabe añadir que el profesor de una segunda lengua a distancia mantiene los cometidos 
relacionados con el objeto de estudio y es un interlocutor 'ideal' en línea, por la calidad tanto de su ínput como de su feedback.

Naturalmente, existen muchas implementaciones de la metodología a distancia. La enseñanza a distancia abarca múltiples combinaciones entre materiales multimodales y actividades síncronas y asíncronas, que incluyen, entre otras, clases magistrales grabadas, debates, proyectos colaborativos, etc. Con la amplia disponibilidad de acceso fiable y robusto a Internet, ha aumentado inevitablemente el uso de recursos en línea, no sólo textuales, sino también de audio y video para el aprendizaje de las segundas lenguas, siendo el inglés la lengua de la que más recursos hay disponibles. El e-Learning ha proporcionado una oportunidad de armar y estructurar cursos alrededor de estos recursos, junto con herramientas de comunicación como los chats y los foros. Precisamente, una línea de investigación muy vinculada con el aprendizaje de segundas lenguas es el aprendizaje social. Chinnery (2008) definió un conjunto de características sobre la forma óptima de aprender lenguas en línea que incluye el componente social: productiva, informativa, colaborativa, comunicativa y acumulativa. El término aprendizaje social, en su sentido más amplio, hace referencia a un tipo de aprendizaje abierto, no estructurado, y no está necesariamente ceñido a la Teoría de Aprendizaje Social (Social Learning Theory; Bandura, 1977). Hoy en día se usa con frecuencia en relación con las redes sociales que forman parte de la Web 2.0 (tema que ha sido abordado en la literatura desde perspectivas complementarias en trabajos como Brown y Adler, 2008; Prensky, 2009), pero sigue ligado a la idea de aprender como proceso cognitivo que tiene lugar en un contexto social, observando, imitando e interaccionando, comportamientos, actitudes y modelos ajenos. Como puede verse, en el aprendizaje social se integran elementos de la teoría cognitiva del aprendizaje, centrada en los factores cognitivos, y el conductismo, que se basa en la idea de respuestas a estímulos del entorno.

Una linéa de investigación ligada al concepto de aprendizaje social es la de ciencia ciudadana basada en el efecto de la larga cola (del inglés long tail effect; Anderson, 2006), que tiene lugar en la Web 2.0 y está basada en una arquitectura de participantes donde el conocimiento reside en la comunidad (es decir, donde los usuarios de una comunidad aprenden a base de su interacción con otros miembros que, en principio, pueden o no saber más que ellos sobre una cuestión concreta, pero a partir del intercambio de mensajes en el seno de la comunidad o con la ayuda de recursos externos, se van negociando significados y construyendo o redefiniendo su conocimiento, explicándolos más adelante a otras personas y así sucesivamente). Como cabía esperar, no todos los estudiantes se muestran a favor de llevar a cabo su aprendizaje de forma colaborativa. Existen estudiantes que muestran filtros afectivos muy altos y no participan abiertamente en comunidades (Leach, 2012). Otros sencillamente tienen un procesamiento mental abstracto y han manifestado que, desde su perspectiva, las oportunidades de aprendizaje social son interesante, solo si no están orientadas hacia el trabajo colaborativo, ya que estiman que este consume demasiado tiempo que no se ve rentabilizado por la ganancia cognitiva (Chiriac, 2014).

Los MOOC representan un ejemplo puntero de educación abierta, aunque no es la única forma en que esta puede materializarse (grupos de discusión en línea [bulletin boards], comunidades en línea, redes sociales, etc.). El aprendizaje abierto no tiene una definición única, pero se refiere por lo general a una modalidad que se adopta a partir de la detección de necesidades del estudiante según las percibe este; es impartida en formatos relacionados con las comunidades de aprendizaje, los MOOC y el uso de recursos educativos abiertos y suele tener lugar sin condicionantes legales ni económicos, más allá de los programas formales reglados de las instituciones educativas. La ausencia de dichos condicionantes suele tener como consecuencia que los grupos de estudiantes son extraordinariamente numerosos, lo cual justifica el frecuente calificativo de "masivo" en relación con los cursos y las comunidades de aprendizaje abierto.

La aplicación del aprendizaje social, abierto y masivo a las segundas lenguas es reciente y en su investigación destaca tanto casos de éxito como retos por resolver (Barcena, Martín-Monje \& Read, 2015). Un problema clave aquí es que el desarrollo de las capacidades necesarias para el uso eficaz de una segunda lengua no es un proceso meramente orientado al conocimiento, sino que requiere una considerable interacción con otros interlocutores (preferentemente, pero no necesariamente, nativos). Las oportunidades para que se lleve a cabo tal comunicación en el 
aprendizaje social, abierto y masivo existen, pero están limitadas por la naturaleza actual del diseño de aprendizaje, que oscila entre una interacción muy estrechamente controlada relacionada con una tarea dada, donde los estudiantes más y mejor regulados pueden impacientarse, o una interacción completamente desestructurada donde los estudiantes menos competentes pueden sentirse perdidos o solos y la obligación por parte de los estudiantes de estar sentados estáticos frente a sus ordenadores.

Casi todos los retos de los LMOOC se comparten con el resto de estos cursos ya que se trata de cuestiones trasversales, como hallar un modelo de calidad, tras un periodo de intentos frustrados (Jansen, Rosewell y Kear, 2017). Este tema tiene dos vertientes: una académica ligada con el desequilibrio en la ratio profesores: estudiantes y las formas de administrar feedback y atender a la diversidad. La segunda vertiente de la calidad de estos cursos está ligada al bajo índice de éxito, el bajo porcentaje de estudiantes que solicitan acreditación (de pago), la sostenibilidad de los cursos y el modelo de negocio para las instituciones. Se están produciendo avances prometedores y al periodo de euforia inicial, durante la implantación de los primeros programas de enseñanza abierta y la puesta en marcha de las consiguientes plataformas en algunas universidades siguió otro periodo de decepción ante los altos índices de abandono, los altos costes de producción y la falta de solicitudes de acreditación que, si bien no llegaron a causar el cierre de estas iniciativas, sí causaron una reducción en las partidas que se estaban invirtiendo en su infraestructura, gestión y publicidad. Con el tiempo, las universidades han comenzado a ver beneficios alternativos a los económicos por vía de las acreditaciones, como los cursos cero, los módulos altamente especializados y la visibilidad de la calidad académica global de la institución que pueden proyectar.

La tecnología móvil se está convirtiendo en una parte estándar de la vida y se estima que para 2020 el $80 \%$ de todos los adultos en el mundo llevará encima algún dispositivo de este tipo (Evans, 2014). Muchas personas hoy en día no tienen un ordenador, pero sí un dispositivo móvil, por lo que desde él se puede ofrecer la oportunidad a un gran número de personas de aprender otra lengua. El aprendizaje móvil no consiste únicamente en el uso de un dispositivo concreto en lugar de un ordenador de sobremesa, sino en los distintos tipos de oportunidades de aprendizaje que surgen cuando se emplean estratégicamente las diferentes funcionalidades del dispositivo y se incorporan los contextos culturales, situacionales e interpersonales del usuario a la configuración de la actividad, particularmente cuando el dispositivo está conectado a Internet. Potenciar el rol de los dispositivos móviles ayuda, además, a difuminar aún más los límites entre las actividades de aprendizaje estándar y lo que se ha venido a denominar vida digital (Read y Barcena, 2015). Esto hace referencia a un aumento de flexibilidad en la forma en que las personas combinan su aprendizaje con sus actividades cotidianas, como tener una página en Facebook en inglés para interaccionar con nuestros allegados extranjeros, lo que también abre la posibilidad de alterar positivamente la forma de alcanzar sus objetivos de aprendizaje, al contar en este caso con una fuente continua de práctica comunicativa auténtica en línea.

La aplicación de la tecnología móvil al aprendizaje de una segunda lengua tiene ventajas e inconvenientes. En cuanto a las primeras, en primer lugar, permite a los usuarios acceder a las actividades de aprendizaje en cualquier lugar y hora, lo cual es cómodo y les permite aprovechar al máximo su tiempo libre o cualquier rato que quieran o puedan utilizar para aprender. En segundo lugar, esta tecnología lleva de una forma "natural" al aprendizaje social, ya que los usuarios geográficamente separados pueden colaborar con sus compañeros para interaccionar, discutir y aprender. En tercer lugar, ampliar o extender el aprendizaje a actividades que requieren el uso de dispositivos móviles puede atraer y mantener el interés de los usuarios, ya que les brinda oportunidades de conectarse a nivel social y la posibilidad de alejarse de los ambientes "academicistas", a veces limitadores. En cuarto lugar, los dispositivos móviles permiten a los estudiantes trabajar a su propio ritmo y revisar los materiales si/cuando/donde quieran. En quinto y último lugar, estos dispositivos apoyan diferentes estilos de aprendizaje, posibilitando el aprendizaje mediante la lectura de textos, el visionado de videos, la escucha de podcasts y la realización de tareas o proyectos en Internet, por poner algunos ejemplos. 
En cuanto a los inconvenientes, en primer lugar, cabe destacar que los problemas de conectividad pueden limitar la capacidad del aprendiz para estudiar en línea. En segundo lugar, el reducido tamaño de la pantalla puede molestar la vista del usuario después de un período prolongado de tiempo. En tercer lugar, el tipo de dispositivo y su sistema operativo subyacente condicionan los materiales y recursos en línea que se pueden utilizar. Los dispositivos más flexibles suelen ser caros, al igual que los planes de datos mensuales que deben abonarse para obtener acceso a Internet. En cuarto y último lugar, los usuarios pueden distraerse si están tratando de trabajar con los materiales de un curso y reciben llamadas telefónicas o WhatsApp. Estas limitaciones, junto con el gran número de apps y el modo ad hoc en el que se suelen utilizar, han impedido que MALL desempeñe hasta la fecha un papel más prominente en el ámbito de las segundas lenguas.

Existe un cuerpo de publicaciones de investigación y proyectos en los que se evidencia que el aprendizaje de segundas lenguas a distancia es más efectivo cuando se utiliza un enfoque espiral que alterna no solo el aprendizaje individual con el social, sino el tutelado con el autodirigido y el monitorizado y el mecánico (Bárcena, 2009; Martín-Monje y Talaván, 2014; Read y Bárcena, 2014). Este planteamiento es coherente con muchos escenarios que se pueden hallar en las instituciones de educación superior y en particular de educación a distancia.

\section{La enseñanza de una segunda lengua a distancia en línea}

Existen múltiples definiciones y análisis de cómo el factor distancia afecta la forma en que los estudiantes desarrollan sus competencias lingüístico-comunicativas. La propuesta más citada es la de Keegan (1996), que define tres características de la educación a distancia que son clave para la enseñanza y el aprendizaje de las lenguas. En primer lugar, la separación física de profesores y estudiantes, que en sí misma constituye un reto; en segundo lugar, el papel de la tecnología, que proporciona la comunicación bidireccional y el acceso al contenido educativo de forma flexible e inmediata y en tercer lugar, la estructura de estos estudios, que se distingue de la de otros por su sistematicidad. Aunque mucho se ha debatido desde esta caracterización, casi dos décadas después, el modelo de Keegan está ampliamente referenciado en la literatura sobre el aprendizaje de lenguas a distancia (por ejemplo, Harrell, 2008; Andrade \& Bunker, 2009).

La evolución de la enseñanza y el aprendizaje de las lenguas a distancia refleja tanto el desarrollo de la tecnología educativa como los avances en materia de lingüística teórica y aplicada. Su estado actual es el resultado de esta amalgama de factores y de las tensiones entre el hecho lingüístico, los requisitos de orden didáctico tanto para el profesor como para el estudiante y la viabilidad tecnológica. Según Wang y Sun (2001), la enseñanza de lenguas a distancia ha pasado por cuatro generaciones no consecutivas, donde la primera generación está esencialmente basada en la impresión de cursos por correspondencia y se argumenta que han durado casi un siglo (el primero apareció en 1728) y todavía está presente en muchas partes del mundo. La segunda generación (que apareció en la década de 1970) combina tecnologías sencillas de multimedia y de difusión, como audio y video y el uso del teléfono. Su surgimiento representó una revolución en la presentación de los materiales de aprendizaje, pero ha demostrado ser limitada en términos de la manera en que los estudiantes pueden interaccionar y recibir feedback por parte de sus profesores. La tercera generación (que apareció a principios de los años 90) trajo consigo CD-ROM interactivos, la disponibilidad de materiales de cursos en línea y el uso de correo electrónico y foros para la discusión y la comunicación en general asistida por ordenador (Computer Mediated Communication o CMC). CMC fue clave para esta generación y la evolución de la enseñanza a distancia, conduciendo a un mayor volumen de interacción estudiante-estudiante, estudiante-profesor y estudiante-hablante nativo (White, 2006). A pesar de que posteriormente sería criticado por su falta de fundamentación y rigor pedagógicos entre otros aspectos (Oppenheimer, 1997), fue en esta generación y en la posterior cuando el término tecnologías de la información y la comunicación (o, más comúnmente, su acrónimo TIC) empezó a ser utilizado incesantemente en relación con el proceso de aprendizaje de lenguas. La cuarta y última generación (que apareció poco después, a mediados de esa misma década) refleja ya la madurez de Internet y la Web y es cuando se incorpora la interacción síncrona, como el chat por 
Internet, las videoconferencias y las aulas y las comunidades virtuales. Esta generación proporcionó a los estudiantes exposición y práctica de la lengua y cultura metas mediadas por la tecnología que han coincidido, posiblemente no por azar, con una evolución de las formas de comunicación tanto en la esfera social como la ocupacional, que tienen lugar cada vez más a distancia y a través de la tecnología.

Existe un considerable volumen de investigación que demuestra que la educación a distancia en general puede producir resultados similares, si no superiores, a las aulas presenciales (por ejemplo, Hanover Research, 2011). Algunos estudios específicos se han centrado en las lenguas y las características que hacen que los cursos en línea sean eficaces para esta materia. Don (2005) utilizó los datos recogidos de un cuestionario dado a los estudiantes de un curso de español en línea, identificando los requisitos que debe cumplir un curso eficaz de segundas lenguas a distancia: instrucciones y documentación claras, comunicación efectiva entre los estudiantes y entre los estudiantes y los profesores, enfoque paralelo y compensado en las cuatro competencias lingüísticas (lectura, escritura, escucha y expresión oral) y materiales de audio. Coryell y Chlup (2007) también usaron datos de cuestionarios obtenidos de estudiantes de inglés como segunda lengua en los Estados Unidos usando distintos programas de software, sitios web y plataformas de aprendizaje, concluyendo que los elementos clave para el buen funcionamiento de un curso incluyen la formación de los profesores, el grado de colaboración promovida y la naturaleza de la instrucción individual y el apoyo brindados. Vorobel y Kim (2012) han analizado artículos de 2005 a 2010 sobre estudios que a su vez han comparado estudios de lenguas presenciales y a distancia. Los textos sorprendentemente incluyen una proporción igual de enfoques metodológicos cualitativos y cuantitativos y logros comparables en términos de la eficacia del curso en el alcance de sus objetivos, la efectividad de su estructura, la satisfacción del estudiante, la calidad de la pedagogía utilizada, la presencia de colaboración y la evaluación. Así pues, mientras que los resultados presentes en la literatura apoyan el uso de la enseñanza de lenguas a distancia sobre su equivalente presencial siempre que sea necesario o incluso posible, cabe abogar por más investigación que siga depurando estas conclusiones y atendiendo los grandes retos de aquella, como son la participación y el abandono.

\section{El enfoque social}

El aprendizaje social es aquel que tiene lugar a través de la interacción entre los compañeros. Puede o no conducir a un cambio en las actitudes y/o el comportamiento de los sujetos, pero se considera en general que está intrínsecamente unido al paradigma socioconstructivista del aprendizaje, que, aún siendo un concepto general o interdisciplinar, es idóneo para materias como la lengua y la comunicación, cuyo desempeño es eminentemente interpersonal (Wijayanti, 2017). Para ser considerado social, un proceso debe, en primer lugar, demostrar que se ha producido un cambio en el conocimiento o el entendimiento de los individuos involucrados y, en segundo lugar, demostrar que este cambio va más allá del individuo y su profesor o materiales y se sitúa dentro de unidades sociales o comunidades de práctica más amplias a través de lo que se considera como relaciones sociales, que habitualmente se sitúan entre las personales o privadas y las ocupacionales (Reed, Evely, Cundill, Fazey, Glass, Laing, Newig, Parrish, Prell, Raymond \& Stringer, 2010).

El aprendizaje social se entronca históricamente con el CMC (Turoff, 1991) de los años 80 y 90. De la misma forma que se han alabado unánimemente las iniciativas de proyectos colaborativos entre grupos de nativos de distintas lenguas, etc. (Thorne \& Payne, 2005), también han surgido críticas relacionadas con la informalidad o desestructuración del proceso formativo, que lleva a los estudiantes a ser menos cultos, humanistas y críticos en su pensamiento (Oppenheimer, 1997), además de, en alguna ocasión, a llegar a conclusiones erróneas. Hay que señalar que todavía queda mucho por investigar sobre el equilibrio idóneo entre el aprendizaje informal (representado por la versión más extrema del aprendizaje social) y el formal o convencional (basado por lo general en un programa didáctico preestablecido por el docente $\mathrm{e}$ impartido en el aula y/o por ordenador). 
Sin embargo, la gran mayoría de estos cursos se ejecutan en plataformas institucionales "cerradas", donde el acceso abierto general no es posible. La Web ha ido evolucionando gradualmente hasta convertirse en una gigantesca infraestructura social donde la mayoría de las herramientas y servicios tienen un componente que permite que las personas puedan discutir o dar su opinión sobre lo que están leyendo/usando o proporcionar enlaces a las principales redes sociales, donde se puede hacer esto mismo. La popularidad de esta infraestructura ha llevado a las personas a querer aplicarlo para cubrir sus necesidades de aprendizaje. Poco a poco, los cursos en línea están empezando a alejarse de las plataformas cerradas acercándose a las abiertas, donde la interacción social entre los estudiantes se ha convertido en un elemento clave en el proceso de aprendizaje. A medida que las personas toman conciencia de la existencia de tales cursos, el número de sus estudiantes ha aumentado drásticamente. Es así como el aprendizaje social, abierto y masivo se ha convertido en un fenómeno educativo que está recibiendo una atención creciente por parte de la comunidad investigadora (Gea, Montes, Rojas, Marín, Cañas, Blanco \& Gutierrez, 2013). Actualmente, debido a su popularidad, la mayor parte del aprendizaje abierto tiene lugar en forma de cursos en línea abiertos y masivos, que están libremente disponibles desde plataformas específicas que normalmente no funcionan bien en los smartphones o tabletas. Por lo tanto, se debe intentar optimizar las ventajas individuales de cada una de estas modalidades didácticas en un marco fundamentado e integrado y demostrar que el aprendizaje social y abierto de lenguas, junto con la tecnología móvil, no representan únicamente formas mejores y más motivadoras de llevar a cabo el aprendizaje de una segunda lengua, sino que son cruciales porque responden a las nuevas formas de comunicación del siglo XXI.

Bandura (1977) formuló cuatro principios teóricos que se encuentran en el núcleo del aprendizaje social: en primer lugar, la atención. Los seres humanos son más propensos a aprender algo si estamos enfocado en ello, es decir, si lo hacemos el foco de su atención. En ese sentido, los contextos sociales ayudan a reforzar dicho foco. En segundo lugar, la retención. Las personas aprenden a través de la internalización de la información en su memoria. Esa información se recuerda o recupera más adelante, cuando se debe responder a una situación similar a la situación en la que se aprendió la información por primera vez. En tercer lugar, la reproducción. Las personas reproducen información previamente aprendida (comportamiento, habilidades, conocimiento, etc.) cuando se requiere y la práctica a través de ensayos físicos y mentales a menudo mejora las respuestas. En cuarto lugar, la motivación. A menudo la motivación necesaria para hacer algo se origina en la propia observación de que alguien es recompensado o castigado por algo que ha hecho o dicho. Esto, por lo general, motiva a las personas más adelante a hacer, o evitar hacer, lo mismo.

El modelado social tiene una aplicación directa en la educación. Si los estudiantes ven consecuencias positivas de un tipo particular de comportamiento (por ejemplo, un compañero que aclara todas sus dudas a través de preguntas), son más propensos a repetir ese comportamiento ellos mismos. Por el contrario, si las consecuencias son negativas, son menos propensos a realizar ese comportamiento. Los contextos nuevos y únicos captan a menudo la atención de los estudiantes, y pueden destacarse en la memoria. Los estudiantes están más motivados a prestar atención si ven que otros a su alrededor también prestan atención. Otra reflexión ligada a esta teoría es que los estudiantes desarrollan su autoestima y la conciencia de sus límites a través de la comparación y la retroalimentación constructivas. Además, existen estudios que demuestran que estos roles sociales que se crean (como el estudiante coordinador, monitor, etc.) son muy positivos tanto para los estudiantes con problemas, que son más abiertos y participativos ante otros compañeros suyos que ante el profesor, para los estudiantes avanzados, que encuentran la tarea de asistir a sus compañeros no solo gratificante, sino una oportunidad de depurar y ratificar sus propios conocimientos y, evidentemente, para el profesor, que ve aliviada una parte de su carga, máxime en cursos masificados.

Los investigadores han redefinido desde entonces la formación social de formas múltiples y superpuestas y a menudo se confunde la formación social con las condiciones y métodos necesarios para facilitar el aprendizaje social o sus potenciales resultados. Es importante distinguir el aprendizaje social como concepto de las condiciones o métodos que pueden facilitar el 
aprendizaje social y los resultados potenciales de los procesos de aprendizaje social. El aprendizaje social no es privativo del aula física y ha experimentado un enorme crecimiento con la llegada de la web 2.0, las plataformas educativas como Moodle, Sakai o aLF y, por supuesto, las redes sociales. El concepto de aprendizaje social excede el de tutorización, en el que la interacción se realiza de forma unívoca entre el experto (normalmente un profesor, pero en ocasiones también un estudiante avanzado) y el aprendiz, ejerciendo el primero como entrenador, dinamizador, mentor y consejero. Este patrón es muy útil para tareas de consultoría privada, cuando el estudiante requiere privacidad por cualquier razón, incluyendo la creencia de que probablemente solo sea pertinente para él o cualquier otro motivo de índole personal o psicológica.

El conectivismo de Siemens (2005) y Downes (2008) ha hecho avanzar la teoría social del aprendizaje de una forma que es consistente con la realidad del siglo XXI, una realidad digital cuyo factor diferencial respecto a tiempos pasados es la tecnología y las redes. El rol que desempeñan en las vidas humanas $y$, en concreto, en el aprendizaje han cambiado el conocimiento de ser algo que entra en la mente humana del aprendiz, se acumula y, en principio, permanece, a residir en una diversidad cambiante y a requerir la continua (re-)creación y consolidación de conexiones multidireccionales, humanas o no, para subsistir y amplificarse. Así pues, otros formatos de interacción académica comunes hoy en día en la educación a distancia incluyen los pequeños grupos de trabajo, las comunidades cerradas (por ejemplo, de cursos virtuales numerosos de estudiantes en una plataforma institucional) y las comunidades abiertas (por ejemplo, las plataformas de sensibilización colectiva; Sestini, 2012). Todas ellas tienen en común no solo el factor humano como recurso de aprendizaje sino la necesidad de contar con herramientas tecnológicas sociales, tales como Edmodo (que proporciona un lugar seguro para conectarse profesor y estudiantes y colaborar, compartir contenidos, acceder a las tareas, etc.), Edublogs (que permite la creación y gestión de blogs de profesor y estudiantes), Elgg (un motor abierto que proporciona un marco robusto para construir todo tipo de entornos sociales), ePals (plataforma conectada de forma segura con aulas de cualquier punto del mundo para emprender actividades de aprendizaje colaborativo basado en proyectos) y un largo etcétera.

\section{El enfoque abierto}

A pesar de lo que algunos medios de comunicación han parecido transmitir a la sociedad, los MOOC no surgieron espontáneamente como una "revolución educativa", sino que representan una evolución natural de los recursos educativos en abierto (OER, según las siglas en inglés) (Bárcena, 2009). Los recursos educativos en abierto existen desde hace casi tanto tiempo como el propio Internet, algunos de los cuales con una enorme popularidad por su calidad como los de la $\mathrm{BBC}^{2}$ y el British Council ${ }^{3}$ para inglés. Sin embargo, no fue hasta 2008 cuando Dave Cormier empleó explícitamente el término $M O O C$ para referirse a un curso en línea abierto y masivo (Watters, 2012). Downes (2012) sostendría posteriormente que los MOOC tienen un potencial didáctico sin precedentes en cuanto que permiten a un gran número de personas combinar las ventajas de los contenidos abiertos con el concepto del aprendizaje - la formación y el desarrollo personal - abierto.

Esta modalidad educativa intenta promover el aprendizaje para un gran número de personas con un interés compartido, eliminando las trabas iniciales de acceso y asistencia y, en algunos casos, se ofrecen créditos y/o certificados a muy bajo coste al final del curso. No es de extrañar, por lo tanto, que a pesar del conflicto de intereses con las prioridades de las instituciones educativas formales y de las muchas críticas que se plantean al respecto en la literatura (Romeo, 2012; Jackson, 2013), los MOOC están teniendo un impacto significativo en la comunidad educativa en línea (con cientos de miles de personas realizando estos cursos en todo el mundo

\footnotetext{
${ }^{2}$ http://www.bbc.co.uk/languages/

${ }^{3}$ https://www.britishcouncil.org/english
} 
ahora mismo) y la comunidad investigadora internacional (con proyectos como ECO4 y HOME5, entre muchos otros). De hecho, han atraído a la atención pública y han sido aclamados como un "fenómeno educativo" (Pappano, 2012). 2012 fue llamado "año del MOOC" por el New York Times porque surgió con enorme vitalidad en las universidades norteamericanas de la Ivy League y el momento era idóneo para que aparecieran (Daniel, 2012), por múltiples razones no solo académicas, sino socioeconómicas y demográficas. En la actualidad, a pesar de algunas expectativas insatisfechas y las bajas tasas de finalización, prevalece un considerable interés en el campo, como lo demuestra el creciente número de estos cursos y sus seguidores en todo el mundo.

El número de cursos y plataformas se ha multiplicado desde su creación y el número de inscripciones de estudiantes sigue siendo muy alto ${ }^{6}\left(\mathrm{CSEV}^{7}\right.$, com. pers.). Además, en los últimos años se han celebrado congresos que han tenido como foco los MOOC, como TISLID'14 y 18 y la serie anual EMOOC (European MOOCs Stakeholders Summit). Son varios los factores que se han combinado para que los MOOC, en general, y los LMOOC, en particular, sean posibles y populares. En primer lugar, el acceso generalizado a la Web se ha convertido en una realidad, incluso en muchas de las partes desfavorecidas del mundo (aunque no para todos). En segundo lugar, a medida que aumentan las personas que quieren estudiar, posiblemente hasta más de 100 millones para 2020, será imposible atender a tal demanda en universidades estándar.

En tercer lugar, unido a lo anterior, un gran porcentaje de estas personas no es (ni se prevé que sea a corto plazo) lo suficientemente afortunada para poder pagar las tasas de matriculación habituales (ni, por supuesto, las de universidades de élite como las de la Ivy League, cuyas cuotas de ingreso superan con creces lo que la mayoría se puede permitir). En cuarto lugar, aunque el dinero no fuera una traba, no hay suficiente espacio físico para el aumento en el número de estudiantes que se prevé en los próximos años y más y más campus tendrían que ser construidos incesantemente. No deja de ser una ironía que a medida que aumenta la demanda de educación superior, la competencia entre universidades es cada vez mayor para los estudiantes de pago. Como consecuencia, la división socioeconómica está creciendo. El fenómeno MOOC parece haber surgido de la interrelación de todos estos factores. Además, el impulso inicial de esta iniciativa proviene de prestigiosas universidades que desean atraer a más y mejores estudiantes y encontrar nuevos modelos de negocio con ellos. Desde esta perspectiva, los MOOC representan un potente recurso de marketing de la calidad académica de las instituciones que los ofertan.

Una modalidad tan compleja no podría existir sin retos, incluyendo el tamaño de la masa estudiantil, que dificultan su manejo y gestión (por ejemplo, cómo proporcionar retroalimentación y andamiaje), los problemas de atribución de la autoría de la evaluación y el alto grado de deserción estudiantil (Read, 2014), entre otros. Además, Bárcena y Martín-Monje (2014) han discutido también sus dificultades, como el papel cambiante de los docentes en los LMOOC (donde pasan de ser instructores a facilitadores ${ }^{8}$, por lo que no pueden interaccionar de manera personalizada con la mayoría de los estudiantes inscritos), el mencionado problema de cómo proporcionar una retroalimentación eficaz con una ratio tan desequilibrada profesor-alumno y las dificultades de gestionar un grupo altamente heterogéneo de estudiantes, compuesto por individuos con diferentes niveles de competencias lingüístico-comunicativas en el caso de los LMOOC. Sin embargo, el aprendizaje de una segunda lengua es un proceso eminentemente práctico y dinámico, y como tal, queda en el centro de la escala de idoneidad para esta modalidad didáctica abierta. Esto requiere el desarrollo de un entramado de capacidades (competencias, habilidades y datos) que deben ir sutilmente entrelazados a lo largo del proceso de aprendizaje (Bárcena, 2009). Ello supone tanto la implicación cognitiva (usando habilidades mentales de alto

\footnotetext{
${ }^{4}$ http://ecolearning.eu

${ }^{5} \mathrm{http}: / /$ home.eadtu.eu

${ }^{6} \mathrm{https} / / /$ unedabierta.uned.es/catalogo/

${ }^{7}$ Centro Superior para la Enseñanza Virtual (UNED).

8 Un facilitador es un orientador del aprendizaje y entre sus cometidos está el de proponer actitudes y procedimientos que promuevan la interacción con los contenidos del curso de que se trate.
} 
orden) como la interacción social con hablantes más o menos competentes de la lengua meta (Read, Bárcena, y Rodrigo, 2010).

La experiencia que están adquiriendo los académicos trabajando en el diseño y elaboración de LMOOC está refinando el concepto empíricamente, discriminando lo que funciona mejor, como el promedio de horas de un curso para que sea útil y atractivo, la definición de rasgos prototípicos de los estudiantes que puedan considerarse para la toma de decisiones sobre el diseño del curso o la tecnología de apoyo más adecuada.

Sin embargo, independientemente de la confusión conceptual (y terminológica) relacionada con estos cursos, y los problemas y retos asociados, las cifras de inscripción y las encuestas de satisfacción muestran que son populares entre los estudiantes (Martín-Monje, Bárcena, y Read, 2013). Se puede argumentar que la popularidad proviene en parte de la falta de coste asociado y de la flexibilidad de acceso y compromiso que ofrecen. A diferencia de otras iniciativas relacionadas con los OER, el hecho de que se trate de una oferta completa y guiada de aprendizaje y la orientación social de la mayoría de estos cursos también resultan estimulantes y gratificantes para los estudiantes, sin olvidar el hecho observado de que a las personas les gusta estar en contacto controlado con otras que comparten intereses similares y provienen de diferentes ámbitos geográficos y sociales, algo que también es constatable en las redes sociales (Boyd, Richerson y Henrich, 2011).

\section{El enfoque móvil}

Al mismo tiempo que la naturaleza de los estudios a distancia y en línea ha cambiado y sigue evolucionando hacia nuevas modalidades y formatos, la forma en la que accedemos e interaccionamos en línea también ha cambiado. Ahora vivimos en un "mundo digital" y podemos usar el dispositivo móvil que llevamos en nuestros bolsillos o bolso para tal fin. Desde principios de los 90, los dispositivos móviles se han convertido cada vez más en una parte de la vida cotidiana. Con la llegada del smartphone y el desarrollo de sistemas operativos móviles abiertos como Android, ha habido una "democratización" de tales dispositivos. También la reducción del precio de los dispositivos móviles significa que un sector cada vez mayor de la sociedad ahora tiene acceso a tales teléfonos y tabletas.

No sólo son los dispositivos los que han evolucionado con el tiempo, sino también la tecnología de red subyacente que emplean para la comunicación. Por lo tanto, no es de extrañar que puedan obtenerse servicios de datos móviles de empresas de telecomunicaciones para ofrecer acceso a Internet cuando no se pueda encontrar abierto uno de los cada vez más numerosos puntos de acceso wi-fi). Como señala Evans (2014), "The world is mobile!", afirmación respaldada por multitud de datos como los aproximadamente 5000 billones de smartphones y tabletas vendidos hasta el fin de 2016 (frente a los aproximadamente 3500 billones de ordenadores de sobremesa y portátiles), las 115 billones de descargas de apps en dispositivos iOS y Android, habiendo más de 2 millones de apps iOS y 3 millones de Android. De estas, la categoría más popular es la red social, ya que las personas dedican un tercio del tiempo de uso de su dispositivo móvil a conectar con alguna de ellas. Para estimar el uso de los teléfonos móviles, en la actualidad hay 900 millones de usuarios de WhatsApp y Facebook Messengers y estos han enviado alrededor de 60 billones de mensajes al día durante 2016. En cuanto a otras funcionalidades de estos teléfonos, en este año se han mandado 1,6 billones de fotos y 250 millones de videos al día. También es significativo el hecho de que alrededor de 2500 billones de los usuarios de redes sociales se conecta desde dispositivos móviles (y de los 1800 billones de usuarios regulares de Facebook, 87\% se conecta también desde dispositivos móviles) y la estimación mencionada anteriormente de que para 2020, el $80 \%$ de la población adulta del mundo tendrá un smartphone. 


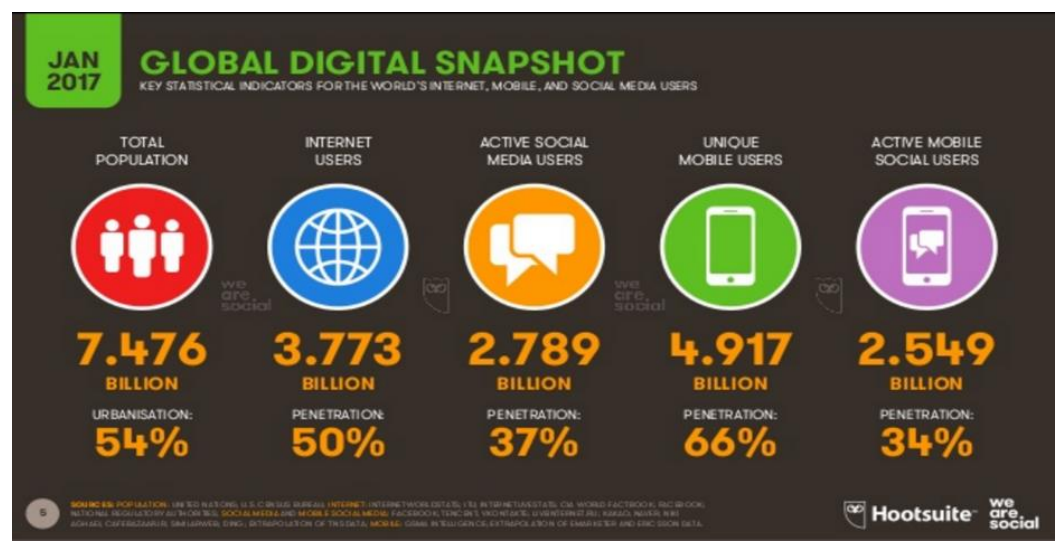

Figura 1. Clasificación de usuarios digitales (Hootsuite, 2017).

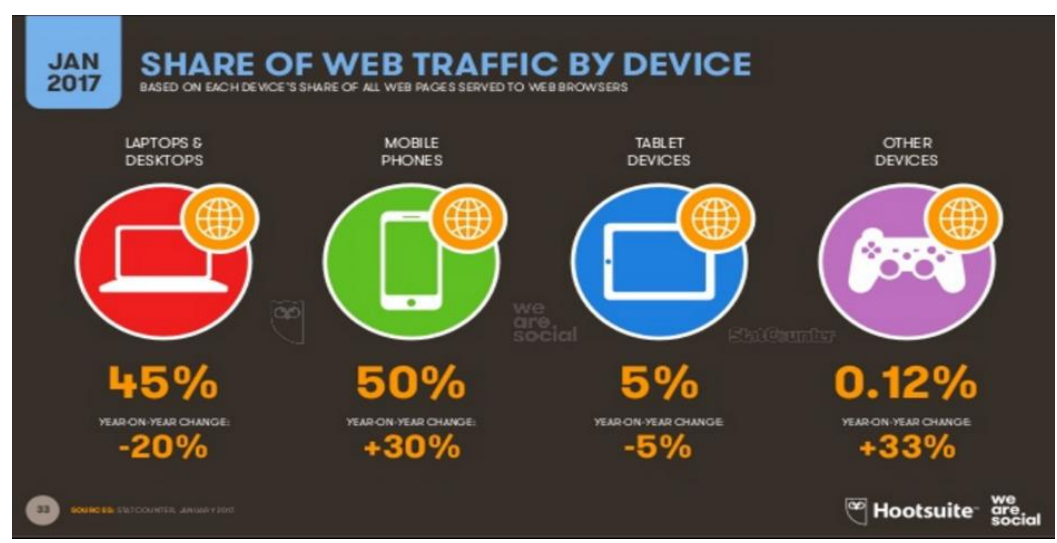

Figura 2. Clasificación de acceso a la web según el dispositivo (Hootsuite, 2017).

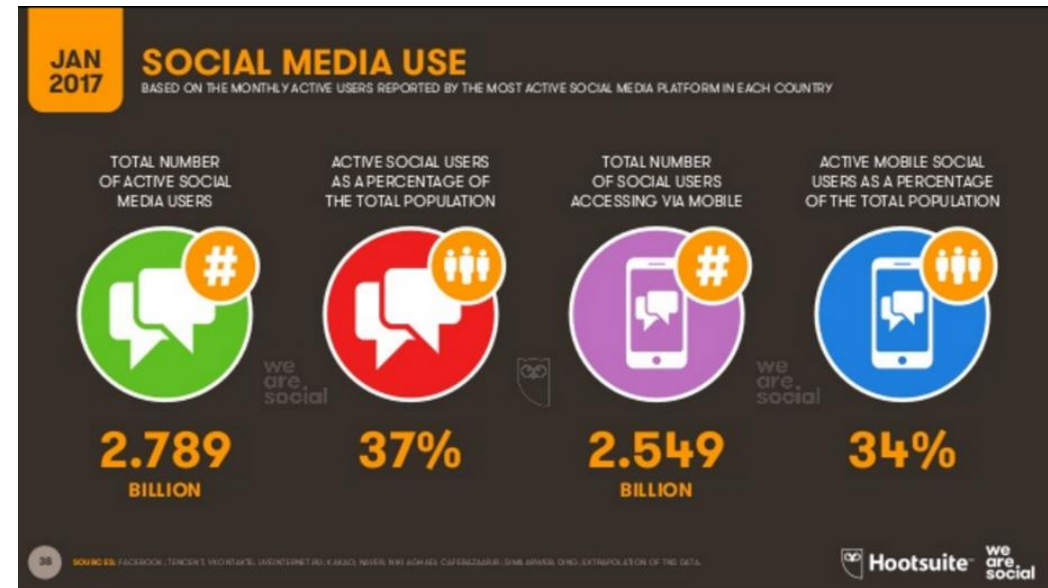

Figura 3. Clasificación de acceso a las redes sociales según el dispositivo (Hootsuite, 2017). 


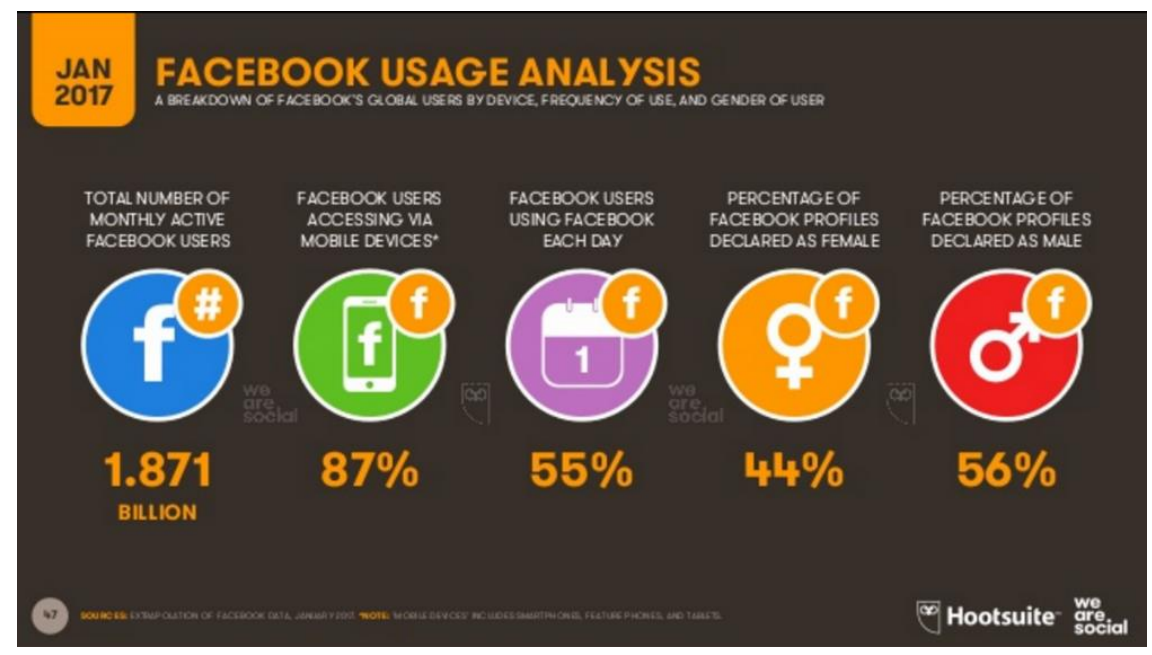

Figura 4. Clasificación de acceso a Facebook según el dispositivo (Hootsuite, 2017).

Al analizar los datos de uso de dispositivos móviles y apps anteriormente señalados, es fácil ver la relevancia que puedan tener los dispositivos móviles en red para el aprendizaje. Es fácil aspirar a que mediante su uso puedan acceder a la formación en una segunda lengua muchísimas personas que no tienen acceso a otros equipos tecnológicos. Sin embargo, si bien las posibilidades de estos dispositivos son infinitas, sin duda queda un largo camino para que desplieguen su pleno potencial. Representan, además, un paso importante hacia el acceso omnipresente a la información y, como tal, condicionarán a corto plazo las formas en que se puede emprender la educación en línea, que los estudiantes están comenzando a explorar. No se trata de que los investigadores traten de atraer a la gente a entornos Web 2.0 desde sus dispositivos móviles, porque ya pueden ver sus beneficios. Esta es una revolución de abajo arriba, donde los propios estudiantes están empujando a sus profesores, no al revés. Este cambio de hábitos refleja un comportamiento humano básico: si estamos acostumbrados a usar una herramienta para algún propósito, inevitablemente trataremos de usarla para otros. Esto quiere decir que, al margen de la predisposición de los estudiantes a emplear los móviles y a las cualidades intrínsecas de estos dispositivos para ello, queda en manos del profesor de la segunda lengua que extienda a través de actos relevantes a su vida cotidiana su tiempo de aprendizaje, más allá de las limitaciones esperables ante el ordenador de sobremesa, y adapte contenidos y métodos a las verdaderas realidades y necesidades de uso.

Una cuestión relacionada es el acceso a contenidos y materiales didácticos a partir de una gama de dispositivos móviles con diferentes características formales y funcionales. Este importante rasgo añadido requiere que los investigadores permitan que el aprendizaje continúe uniformemente entre diferentes dispositivos móviles, es decir, se trata de promover su potencial para que los estudiantes cambien "sin fisuras" de actividades de su vida cotidiana a actividades orientadas a promover el aprendizaje, tratando de reconciliar, por ejemplo, intereses personales o necesidades comunicativas puntuales con el acto de aprendizaje. Ya no es necesario contar con equipos especializados para grabar algo que está sucediendo y compartirlo en línea. Como Looi, Seow, Zhang, So, Chen y Wong (2010) argumentan, la portabilidad y la versatilidad de nuestros dispositivos móviles y el acceso en línea casi ubicuo conllevan importantes posibilidades para un aprendizaje más centrado en el aprendiz.

Esta preocupación por la compatibilidad y la ubicuidad en el aprendizaje móvil ya ha desembocado en la creación de una línea de investigación cuyo objetivo es explorar la forma de garantizar tal uniformidad, que ha recibido el nombre de aprendizaje móvil sin costuras (en adelante MSL, por el término inglés Mobile Seamless Learning). El MSL ha sido caracterizado por Wong y Looi (2011) en términos de diez dimensiones que Wong (2012) ha refinado de la siguiente manera: MSL1 abarca el aprendizaje formal e informal; MSL2 abarca el aprendizaje personalizado y social; MSL3, el aprendizaje a través del tiempo; MSL4, el aprendizaje a través 
de lugares; MSL5, el acceso omnipresente al conocimiento (una combinación del aprendizaje sensible al contexto, realidad aumentada y acceso ubicuo a recursos de aprendizaje en línea); MSL6 abarca mundos físicos y digitales; MSL7, el uso combinado de múltiples tipos de dispositivos (incluyendo tecnologías "estables", como los ordenadores de sobremesa y las pizarras interactivas); MSL8, cambio continuo y rápido entre múltiples tareas de aprendizaje (como la recolección de datos, el análisis y la comunicación); MSL9, síntesis del conocimiento (previo y nuevo, así como múltiples niveles de habilidades de procesamiento mental y/o aprendizaje multidisciplinar) y MSL10, que abarca múltiples modelos de actividad pedagógica (facilitados por los profesores).

La aplicación de MSL a las lenguas ha sido analizada por Wong, Chai, Chin, Hsieh y Liu, (2011) y a los MOOC por de Waard, Keskin y Koutropoulos (2014). Estos últimos señalan que los problemas con estos cursos podrían tratarse en cierta medida extendiendo el contexto de aprendizaje que se asume en cada MOOC estándar para incluir actividades diversas, colaborativas y autodirigidas que reflejen y optimicen la movilidad de los estudiantes. De Waard y otros (2014) argumentan que las diez dimensiones presentadas anteriormente serían más adecuadas para los MOOC (y los MOOC de lenguas) si se modificasen de la siguiente manera: extendiendo MSL1 para incluir el aprendizaje permanente, agregando el aprendizaje colaborativo a MSL2, fusionando MSL3 y MSL4, agregando recursos de aprendizaje basados en la nube a MSL5, combinando actividades de aprendizaje basadas en los mundos físico y digital para MSL6, utilizando múltiples dispositivos (con sus respectivas ventajas) en diferentes contextos para MSL7, añadiendo objetos de aprendizaje compartidos para MSL8 e incorporando aprendizaje centrado en el aprendiz para MSL9 y aprendizaje autorregulado para MSL10. La forma exacta de aplicarse MSL depende del tipo de MOOC (véase más arriba) y también de cada sujeto individual que participa en el curso. Por último, en lugar de considerar el ambicioso objetivo de que todas las actividades se puedan realizar desde todos los dispositivos, es probable que la experiencia demuestre tendencias comunes a realizar ciertas tareas desde ciertos dispositivos y, por lo tanto, determinar su idoneidad representa un objetivo de investigación en sí mismo.

\section{Integración de los enfoques}

En las secciones precedentes se ha argumentado a favor de una serie de enfoques didácticos y procede ver ahora qué cabe esperar de su integración. Las ventajas que se pueden identificar en el aprendizaje social de segundas lenguas en los MOOC incluyen: primero, que las intervenciones escritas de los estudiantes permanecen, lo que permite su análisis posterior; segundo, que se refuerzan necesariamente tanto la interacción como las habilidades interpersonales; tercero, dados los cronogramas más laxos de los cursos abiertos, los estudiantes tienen más tiempo que en otros formatos de aprendizaje para reflexionar sobre las intervenciones de sus compañeros y las suyas propias; cuarto, es relativamente fácil estar en contacto con hablantes nativos (lo cual es de especial relevancia para el aprendizaje de segundas lenguas) y quinto, y posiblemente el más evidente, la interacción social entre los participantes puede constituir el mayor apoyo didáctico en los MOOC del que se benefician, como se comentaba anteriormente, sujeto aprendiz, compañeros y profesor.

Sin embargo, no todos los MOOC son intrínsecamente sociales. Una de las distinciones más importantes encontradas en la literatura con respecto a los MOOC es la que se conoce ya como 'xMOOC vs. cMOOC'. Los primeros son similares a los cursos en línea estándar, pero, por lo general, con un número mucho mayor de estudiantes. Representan la gran mayoría de los cursos existentes, ya que con frecuencia surgen como adaptaciones de cursos anteriores de e-Learning. Los otros, los MOOC conectivistas, se basan en principios de comunidades de aprendizaje con usuarios activos que aportan contenidos y construyen conocimiento, donde las actividades clave son la remezcla, reorientación y co-creación de contenido e interacción (Morrison, 2013). Los xMOOC promueven la diversidad de participantes, en el sentido de que se transmite el mismo mensaje a un grupo tan voluminoso como heterogéneo, mientras que los cMOOC se centran más en la diversidad de los perfiles de participantes y de las formas de desarrollar y distribuir las actividades y los recursos. Los cMOOC no se ejecutan de forma centralizada desde una sola 
plataforma, sino que se distribuyen entre varias, por lo que son difíciles de controlar desde una perspectiva institucional. Si bien tienen la ventaja (para los participantes) de que contienen recursos educativos, prácticas y derechos de autor en abierto, que impiden que el contenido se bloquee en una plataforma o incluso en un individuo, dificultan que una institución determinada controle el proceso educativo. Esto ha sido presentado como una cuestión problemática, al intentar garantizar el desarrollo del estudiante (Brennan, 2014). Sin embargo, como señala Macness (2013), los cMOOC facilitan la inmersión mejor que los xMOOC, aunque requieren que sus participantes sean autorregulados y selectivos, debido a las grandes cantidades de recursos de aprendizaje complejos que se suelen generar en cada curso. La falta de control por parte del estudiante puede conducir a una sobrecarga de información y a la desorientación.

La mayoría de los LMOOC desarrollados hasta la fecha son xMOOC, lo cual posiblemente presenta una limitación fundamental relacionada con la naturaleza misma del aprendizaje de una segunda lengua. Por lo tanto, si se espera que los estudiantes de éxito asuman un papel protagonista en su propio aprendizaje, sobre la base de que el conocimiento es generalmente autoconstruido en lugar de transferido, requieren la oportunidad de construir estrategias y conexiones que sean significativas para ellos en un momento dado, de una manera adaptativa. Sin embargo, dada la naturaleza intrínsecamente social de la comunicación verbal, la negociación del significado, la participación en el trabajo grupal, la prestación de asistencia mutua y la construcción y el intercambio de nuevos conocimientos y habilidades en colaboración con otros han sido ampliamente estudiados y elogiados en la literatura de didáctica de segundas lenguas (Warschauer y Kern, 2000; Read, Bárcena, Traxler \& Kukulska-Hulme, 2017). Aunque la producción de los compañeros contiene por lo habitual errores y la validez de su retroalimentación, por lo tanto, puede no ser siempre completamente fiable, el aprendizaje de lenguas ya no se limita a la idea de la imitación del modelo "perfecto" de un solo profesor (y/o conjunto de materiales). En la actualidad, el objetivo último del aprendizaje de segundas lenguas se acepta, por lo general, que es el desempeño verbal inteligible, empático y efectivo, en un variado conjunto de contextos y situaciones y con diferentes tipos de interlocutores (Consejo de Europa, 2001; National Capital Language Resource Centre, 2004). Por lo tanto, el enfoque de diseño utilizado en el aprendizaje efectivo abierto y social de lenguas debe contener elementos específicos de cMOOC para proporcionar los grados de interacción autónoma necesarios para que los usuarios puedan realmente desarrollar sus capacidades.

Para proporcionar un diseño para el aprendizaje efectivo de lenguas en línea abierto y social, un xMOOC estándar carecería de proactividad e interactividad necesarias para garantizar el desarrollo de las competencias lingüístico-comunicativas que, por el contrario, sí se contempla en los cMOOC. Yeager, Hurley-Dasgupta y Bliss (2013) identifican cuatro tipos de actividades en los cMOOC que pueden incrementar la proactividad de los estudiantes y facilitar la interacción entre ellos: agregación/curación, remezcla, reorientación y distribución. Sin embargo, la aplicación de la filosofía completa de cMOOC (de acuerdo con la cual los estudiantes exploran libremente un gran espacio conceptual de recursos en línea y medios sociales relacionados de una manera casi ad hoc, en gran medida improvisando sus selecciones a medida que avanzan) no se ve necesariamente como la manera más efectiva de progresar. Una vez que los estudiantes hayan obtenido los fundamentos estructurales básicos de la lengua meta (en términos de vocabulario, gramática, pragmática, conocimiento sociocultural, etc.), hay un amplio consenso entre los expertos acerca del hecho de que un enfoque basado progresivamente en el ejercicio de la comunicación es el más apropiado (Knight, 2003).

En los cursos en línea cerrados estándar, hay pocas y restringidas posibilidades de interacción real en la lengua meta. En los LMOOC, por el contrario, el 'factor cuantitativo', es decir, el número potencialmente elevado de estudiantes, asegura suficiente 'masa crítica' que posibilita ínput variado, foros animados, una rica interacción entre compañeros (por ejemplo, para hacer observaciones, responder a dudas), mayor presencia y una distinción más fácil (vía gamificación por karma o sistemas de puntuación similares) de los estudiantes avanzados, destacados o particularmente colaboradores y, relacionado con todo esto, pero más importante aún, mayores posibilidades de generarse ínput válido colectivamente, en el propio seno del grupo. 
Las herramientas y redes sociales en línea, si están correctamente estructuradas y gestionadas, pueden combinar lo mejor del aprendizaje formal, informal y no-formal para aprovechar las posibilidades de desarrollo de competencias lingüístico-comunicativas en una segunda lengua, especialmente las productivas y las interactivas. El aprendizaje abierto y social de lenguas puede potenciar la interacción efectiva de los estudiantes y permitirles comunicarse en la lengua meta, utilizando estrategias (meta-)cognitivas similares a las que emplearían en situaciones comunicativas auténticas (por ejemplo, razonamiento, contraste, indagación, justificación, reflexión, etc.). Esto requiere que todo el proceso esté estructurado y contenga mecanismos interrelacionados que eviten que los estudiantes pierdan su motivación, se desorienten, no rentabilicen su tiempo de estudio o internalicen lengua errónea más allá de lo razonable.

Tecnológicamente, los dispositivos móviles ofrecen tres beneficios para el aprendizaje social y abierto de lenguas, que pueden complementar la experiencia. En primer lugar, que permiten a los estudiantes continuar sus estudios de una manera flexible, aprovechando al máximo el tiempo que tienen disponible a medida que se mueven y trasladan de un lado a otro todos los días. Este acceso en sí mismo promueve la interacción proactiva en sesiones cortas y frecuentes, lo cual es óptimo para las lenguas. No necesitan esperar para tener una computadora a mano, sino que pueden comprobar si, por ejemplo, algún otro compañero ha comentado algo que han dicho en un foro o red social. En segundo lugar, los smartphones modernos proporcionan una forma enriquecida para que los estudiantes interactúen con el mundo que los rodea, grabando sonidos y habla, tomando fotos, obteniendo datos geográficos o lingüísticos, etc. Otros ejemplos incluyen encontrar un determinado tipo de objeto, tomar una foto de él, etiquetar las partes del objeto en la foto y cargarlo en el curso para sus compañeros con el fin de seguir con la misma actividad. En tercer y último lugar, los dispositivos móviles modernos son en sí mismos pequeños pero poderosos ordenadores portátiles basados en una arquitectura extensible de aplicaciones que pueden proporcionar herramientas generales para complementar el aprendizaje social abierto de lenguas, ya sea como parte de un curso o como algún tipo de actividad complementaria (GodwinJones, 2011).

El estado de desarrollo de plataformas MOOC para el despliegue móvil ha sido resumido por de Waard (2014). Algunas plataformas permiten el acceso a algunos de sus recursos desde dispositivos móviles como, por ejemplo, Alison (http://alison.com), EdX (www.edx.org), Khan Academy (www.khanacademy.org) y Udacity (www.udacity.com), mientras que otras como FutureLearn (www.futurelearn.com) y Eliademy (https://eliademy.com) cuentan con cursos que funcionan completamente desde un dispositivo móvil si están conectados a Internet. El enfoque MALL de apps, como el de Canvas Network (www.canvas.net) y Udemy (www.udemy.com), es ligeramente diferente porque, además de proporcionar acceso a una plataforma determinada, la app típica también permite descargar las lecciones para su uso sin conexión. Por ejemplo, la app de Coursera (www.coursera.org) permite a los estudiantes seguir los cursos en los que están matriculados, transmitir o descargar los videos correspondientes, pero hace falta realmente un ordenador para hacer las actividades y entregar las tareas o deberes. Sólo está disponible para las versiones más recientes de iOS y Android, por lo que cualquier estudiante con un dispositivo antiguo o incluso un teléfono nuevo de Windows no puede utilizarlo. Además, dado que no hay una versión diferente de la app para smartphones o tabletas, los usuarios de los primeros pueden sentirse frustrados porque realizar actividades en una tableta es más viable que en un teléfono, ya que tanto las pantallas como los teclados son más grandes y fáciles de usar.

Dada la amplia adopción de dispositivos móviles, es sólo cuestión de tiempo antes de que la mayoría, si no todos los principales proveedores MOOC preparen sus cursos y herramientas relacionadas en formato móvil. En el debate incipiente acerca del tema de la integración de los LMOOC en el formato móvil, se ha presentado el razonamiento de que si hay estudiantes realizando un MOOC intentarán hacerlo desde su dispositivo móvil (que sin duda estarán utilizando con otros fines) para continuar con esta actividad cuando sea posible (Kukulska-Hulme, Traxler y Pettit, 2007). Esta premisa está implícita en gran parte de la investigación que se está llevando a cabo en MALL. La siguiente reflexión es que, dado el hecho de que los estudiantes 
están utilizando sus dispositivos móviles para acceder a los recursos educativos en línea de todos modos, explorar el acceso a LMOOC desde estos dispositivos debe ser el siguiente paso.

Las limitaciones de soporte de los MOOC se están haciendo cada vez más evidentes con los nuevos proyectos colaborativos que están empezando a aparecer, como la asociación EdX con Facebook para el acceso a cursos móviles en África9 ${ }^{9}$. Otras iniciativas más recientes, como Indian My Open Courses, fueron compatibles con móviles desde el principio ${ }^{10}$. Incluso las plataformas MOOC que no admiten el acceso móvil pueden ofrecer contenidos y recursos para su descarga primeramente a un ordenador y luego su transferencia a un dispositivo móvil. También hay que tener en cuenta que, dada la amplia gama de herramientas compatibles con móviles 2.0 que están disponibles en estos días, es cuestionable si una auténtica plataforma MOOC es realmente necesaria para ofrecer aprendizaje social y abierto a los estudiantes estos días. Por ello, puede ser de interés no contar con ninguna plataforma MOOC específica para el aprendizaje abierto y social de lenguas, lo cual es un campo en gran parte sin explorar, ya que la gran mayoría de estos cursos están limitados por las restricciones de plataformas MOOC específicas.

\section{Conclusiones}

Este artículo ha partido de la reflexión de que la enseñanza y el aprendizaje de lenguas a distancia se refieren a las dos caras de un viejo proceso que está adoptando nuevas formas con la continua llegada y distribución de tecnologías nuevas y más sofisticadas que permiten conectar a los estudiantes con herramientas y recursos cada vez más atractivos y adaptativos, así como con otros individuos con intereses similares o complementarios. Estos estudiantes están asistiendo a una sustitución, al menos parcial, de los materiales y procedimientos convencionales del entorno académico a distancia (García Aretio, 2017) por otros en un escenario geográficamente disperso que, pese a ello, no frena el trepidante flujo de información y comunicación interpersonal, de nuevas ocasiones de colaboración y de la adopción de nuevos roles y responsabilidades por parte de todos los actores involucrados. Ante esta realidad en continua evolución, cabe enfatizar el valor de un tipo de aprendizaje horizontal, que tiene lugar eminentemente por contraste y negociación con pares. Este tipo de aprendizaje cuestiona los paradigmas anteriores por imitación de un modelo y los sustituye o amplía con otro que conlleva la compartición, negociación, reorientación, curación y reinterpretación de conocimiento que aflora de una forma distribuida, dinámica y multidireccional en la colectividad. Cabe añadir también, a propósito de las nuevas modalidades de aprendizaje de lenguas en línea, casual, abierta, gamificada, etc., que la tecnología per se no es tan importante como todas las mentes que está abriendo, las actitudes que está cambiando y, por todo ello, las oportunidades de aprendizaje que están surgiendo donde antes no se hubiera contemplado.

En este artículo se ha hecho referencia al concepto de aprendizaje más que al de enseñanza. Esto responde a la influencia de teorías psicopedagógicas como las de Piaget (1947), Vygotsky (1978) y Siemens (2005), que han ido ganando terreno en el área de la educación y cuyo foco ha sido invariablemente el sujeto aprendiente. Sin embargo, debe enfatizarse que dicho foco no supone un detrimento para la relevancia de la figura del profesor sino, bien al contrario, cuanto más eficaz y mejores resultados se obtienen de cualquier proceso de aprendizaje, mayor es el mérito del docente por haberlo diseñado y dirigido con el menor número de carencias, dependencias o debilidades. Por otra parte, cuanto más desequilibrada es la ratio profesorestudiante, más fácilmente constatable es el efecto de la actividad del profesor, particularmente en cursos en línea, donde el estudiante debe poder progresar de forma dinámica y distribuida entre los caminos de aprendizaje y los recursos y actividades puestos a su disposición por el profesor.

Mucho se ha escrito sobre los "nuevos roles del profesor" en los entornos didácticos en línea y sobre su necesidad de formación especializada para incorporarlos a su docencia (McGhee y Kozma, 2007; Elliott, 2011). Al igual que ha sucedido en el resto de las profesiones, el docente

\footnotetext{
${ }^{9} \mathrm{http} / / / \mathrm{blog}$.edx.org/working-facebook-create-socialedu

${ }^{10} \mathrm{http}: / /$ myopencourses.com/
} 
está siempre adaptando sus funciones ante la tecnología que tiene a su disposición para que ambas se complementen sin solapamientos ni lagunas. El de los profesores es uno de los casos claros en los que la tecnología, sobre todo la informática, le ha restado un aparente protagonismo, lo cual se comentaba anteriormente que suele recogerse en la literatura con la rima "from sage on the stage to guide on the side" ("de sabio sobre el escenario, a guía a un lado"; King, 1993). Es común leer el largo listado de roles aparentemente menores que han venido en sustitución del tradicional de instructor: guía, consejero, corrector, revisor, dinamizador, etc. Recientemente, sin embargo, se está empezando a hablar del último rol del profesor, siguiendo la rima anterior: “... to ace in the digital space" ("... a as en el espacio digital"), en la que se vuelve al reconocimiento original, incluso magnificado, reconociendo un dato que arrojan invariablemente las encuestas a los estudiantes en línea: que de todos los elementos que conforman su escenario de aprendizaje, el más valioso es el profesor (Jordano, Bárcena y Talaván, 2013). Esta puntualización coincide en el tiempo con una tendencia a que cada vez más de sus funciones sean compartidas por los estudiantes, como diseñadores de cursos, curadores de contenidos, facilitadores de foros, etc. Debe añadirse que la difusión entre la figura del profesor y del estudiante es aún mayor en los escenarios en línea masivos, donde se constata cada vez más el valor de la masa crítica de conocimiento generado y donde las nuevas técnicas de discriminación positiva pueden servir al profesor para descargar en algunos individuos parte de su carga de trabajo, en una suerte de cascada funcional que está siendo objeto de investigación en la actualidad (Bárcena, MartínMonje \& Read, 2015).

El aprendizaje de una lengua debe tener un componente inherentemente social debido a que esta es ante todo un sistema para la comunicación interpersonal. Para optimizar la interacción entre sujetos que desean aprender una misma lengua, un flujo dinámico y abierto de participantes y contenidos permite alcanzar una masa crítica y heterogénea idónea que está más cerca de reproducir situaciones comunicativas reales y que se genere inteligencia colectiva de una validez superior a la de la suma de las partes que la forman. Aunque en las instituciones superiores de educación a distancia como la UNED ha habido cursos masivos en línea desde hace décadas, el concepto de MOOC, en el sentido de estos cursos en abierto, existe desde hace menos de una década. El surgimiento de los MOOC ha sido un paso importante hacia la educación abierta, incluyendo las segundas lenguas, para el gran número de personas en el mundo que, por diversas razones, no pueden asistir a clases presenciales o participar en cursos comerciales en línea. Sin embargo, dada la diversidad actual de los MOOC, posibilitada por su evolución pedagógica y tecnológica y por el apoyo institucional y político que están recibiendo, es más apropiado pensar en términos de aprendizaje abierto y social de lenguas, ya que hay muchas maneras diferentes de aprovechar la tecnología existente para facilitar el aprendizaje de segundas lenguas que incluyen, pero va más allá, de los formatos MOOC estándar. Por otra parte, los límites entre lo que es un curso y lo que no lo es no siempre son tan claros o relevantes.

Se ha reflexionado largamente acerca del hecho de que los dispositivos móviles pueden complementar el aprendizaje social y abierto de lenguas de tres maneras principales. En primer lugar, pueden proporcionar acceso a recursos de aprendizaje y a otros estudiantes en línea "en cualquier momento-en cualquier lugar" o "en algún momento-en algún lugar" (Hung y Zhang, 2012). Este acceso ofrece la posibilidad de un modelo de interacción y comunicación que reproduce el mundo real de referencia y la modalidad formativa de inmersión de forma inédita hasta ahora, incluyendo el margen de adquisición de conocimientos casual propio de él. En segundo lugar, los dispositivos móviles tienen una amplia gama de sensores que pueden utilizarse para interaccionar con el mundo real. Esto permite a los estudiantes extraer actividades del entorno en línea e incorporarlas a la vida cotidiana. Por otra parte, la información y la interacción pueden grabarse para su posterior reintroducción en el mundo en línea, como recursos para actividades de aprendizaje. Alternativamente, los resultados del aprendizaje en línea pueden utilizarse como recursos de andamiaje para actividades que una persona puede necesitar llevar a cabo en el mundo real. De hecho, se ha hablado en la literatura del doble rol de la tecnología móvil como asistente y como tutor del sujeto que aprende una segunda lengua (Sharples, 2000). En tercer y último lugar, la cultura de apps asociada con los dispositivos móviles proporciona un valioso conjunto de programas para complementar las actividades anteriores realizadas en línea. 
Además de las apps generales que pueden ser útiles para apoyar el aprendizaje, se ha defendido en este artículo que las prácticas de MALL deben estar estrechamente integradas en el aprendizaje abierto y social de lenguas para diversificar y optimizar su potencial. Ya se han realizado algunos trabajos incipientes para aplicar dispositivos móviles a tareas específicas dentro de procesos formativos en línea sociales y abiertos para segundas lenguas, pero no se ha hecho hasta la fecha un uso sistemático y fundamentado de ellos como piedra angular para tal aprendizaje.

Como la vida humana es eminentemente móvil, no es de extrañar que las personas intenten obtener el máximo provecho de las oportunidades que posibilita este tipo de dispositivos mientras se trasladan de un lado a otro, para disponer de los recursos en línea a los que solo hubieran podido acceder previamente permaneciendo estáticos en un único lugar (aparte del hecho constatado de que muchas personas que no disponen de un ordenador de sobremesa, sí cuentan con un dispositivo móvil, normalmente un teléfono). A medida que la tecnología se vuelva más inclusiva, generalizada y ubicua, la fina línea que divide su uso para aprender una lengua de forma autónoma, híbrida o a distancia y apoyar la vida cotidiana se irá haciendo inevitablemente más y más difusa. Por lo tanto, la forma en que las personas usan la tecnología móvil actualmente para acceder a los medios y las redes sociales, o para buscar información relevante para un trabajo que están realizando, se verá extendida para incluir actividades orientadas hacia su aprendizaje y viceversa. Es así como se posibilitaría que el aprendizaje de lenguas móvil, abierto y social abra puertas a nuevas y prometedoras técnicas y estrategias basadas tanto en el contexto como en situaciones y tareas que proporcionen a los usuarios prácticas y recursos de una flexibilidad máxima conocidos como "justo a tiempo" ("just in time"; Kong, Looi, Chan y Huang, 2017), adecuados para muchas vidas modernas. Dado el estado de la investigación en aprendizaje de lenguas en línea, se ha considerado el siguiente paso lógico de exploración hacia una modalidad de aprendizaje ubicuo en un entorno en el que las personas estarán permanentemente en línea, inmersas en una nube de información, lo que dará lugar a oportunidades comunicativas y formativas en la lengua meta en las que participarán "camaleónicamente", a medida que cambien de lugar, dispositivo y quehacer.

\section{Referencias}

Anderson, C. (2006). The long tail: Why the future of business is selling less of more. Nueva York: Hachette Books.

Andrade, M. S., y Bunker, E. L. (2009). Language learning from a distance: A new model for success. Distance Education, 30(1), 47-61. doi: https://doi.org/10.1080/01587910902845956

Bandura, A. (1977). Social Learning Theory. Englewood Cliffs, NJ: Prentice Hall.

Barcena, E. (2009). Designing a Framework for Professional English Distance Learning. En I.K. Brady (Ed.) Helping People to Learn Foreign Languages: Teach-niques and Teachnologies. Murcia: Quaderna Editorial, pp. 89-103.

Barcena, E., Read, T., \& Cuesta Medina, L. (2019). Technological and psychopedagogical innovation in second language blended learning. En A. Palalas (Ed.) The International Handbook of Blended Learning. China Central Radio \& TV University Press Co. Ltd.

Barcena, E., Martín-Monje, E., \& Read, T. (2015). Potentiating the Human Dimension in Language MOOCs. En Proceedings of EMOOCs 2015. Third European MOOCs Stakeholders Summit. Université Catholique de Louvain, 46-54.

Barcena, E., \& Martín-Monje, E. (2014). Language MOOCs: An emerging field. En E. MartínMonje y E. Bárcena (Ed.), pp. 1-15. doi: https://doi.org/10.2478/9783110420067.1

Bates, A.T. (2005). Technology, e-learning and distance education. Londres: Routledge. doi: https://doi.org/10.4324/9780203463772

Boyd, R., Richerson, P.J., \& Henrich, J. (2011). The cultural niche. Why social learning is essential for human adaptation. En J.C. Avise (Ed.) Proceedings of the National Academy of Sicences of the United States of America, 108(Suplemento 2), 10918-10925. doi: https://doi.org/10.1073/pnas.1100290108 
Brennan, K. (2014). In Connectivism, No One Can Hear You Scream: a Guide to Understanding the MOOC Novice. Hybrid Pedagogy. Recuperado de http://www.hybridpedagogy.com/journal/in-connectivism-no-one-can-hear-you-screama-guide-to-understanding-the-mooc-novice/

Brown, J., \& Adler, R. (2008). Minds on Fire: Open Education, the Long Tail, and Learning 2.0. Educause Review, 43(1), 16-32.

Consejo de Europa (2001). Marco Europeo de Referencia para las Lenguas: Aprendizaje, Enseñanza, Evaluación. Madrid: MECD (publicado en español en 2002).

Coryell, J. E., \& Chlup, D. T. (2007). Implementing e-learning components with adult English language learners: Vital factors and lessons learned. Computer Assisted Language Learning, 20(3), 263-278. doi: https://doi.org/10.1080/09588220701489333

Cuesta Medina, L., Anderson, C.E., y McDougald, J.S. (2017). Self-regulation and language teacher training in Colombia. En D.L. Banegas (Ed.), Initial English language teacher education: International perspectives on research, curriculum and practice. Londres: Bloomsbury Academic. doi: https://doi.org/10.5040/9781474294430.0015

Chinnery G. (2008). Biting The Hand That Feeds Me: The Case for e-Learning and Teaching. CALICO Journal, 25(3), 471-481. doi: https://doi.org/10.1558/cj.v25i3.471-481

Chiriac, E.H. (2014). Group work as an incentive for learning - students' experiences of group work. Frontiers in Psychology, 5. doi: https://doi.org/10.3389/fpsyg.2014.00558

Daniel, J. (2012). Making sense of MOOCs: Musings in a Maze of Myth, Paradox and Possibility. Korea National Open University. Academic Partnerships. doi: https://doi.org/10.5334/2012-18

de Waard, I. (2014). Designing \& developing a success MOOC that fits your training environment: platforms. SlideShare. Recuperado de http://www.slideshare.net/ignatia/online-forum-succesfully-integrating-mooc-intraining-environment

de Waard, I., Keskin, N.O., y Koutropoulos, A. (2014). Exploring Future Seamless Learning Research Strands for Massive Open Online Courses. Handbook of Research on Emerging Priorities and Trends in Distance Education: Communication, Pedagogy, and Technology, 201-216. doi: https://doi.org/10.4018/978-1-4666-5162-3.ch014

Don, M. R. (2005). An investigation of the fundamental characteristics in quality online Spanish instruction. CALICO Journal, 285-306. doi: https://doi.org/10.1558/cj.v22i2.285-306

Downes, S. (2012). Half an Hour: Creating the Connectivist Course. Recuperado de http://halfanhour.blogspot.pt/2012/01/creating-connectivist-course.html

Downes, S. (2008). Places to go: Connectivism \& connective knowledge. Innovate: Journal of Online Education, 5(1). Recuperado de http://nsuworks.nova.edu/cgi/viewcontent.cgi?article=1037\&context=innovate

Durán Padilla, A., \& Reséndiz Rodríguez, R. (2007). La distancia de la educación, problemas y desafíos de la educación a distancia. XIX Encuentro Nacional AMIC.

Elliott, J. (Ed.). (2011). Reconstructing teacher education. Londres: Routledge. doi: https://doi.org/10.4324/9780203126066

Ellis, N. (2015). Implicit AND Explicit Language Learning: Their dynamic interface and complexity. En P. Rebuschat (Ed.) Implicit and explicit learning of languages. Amsterdam: John Benjamins, 3-23. doi: https://doi.org/10.1075/sibil.48.01ell

Evans, B. (2014). Mobile is eating the world. Benedict Evans Blog. Recuperado de http://benevans.com/benedictevans/2014/10/28/presentation-mobile-is-eating-the-world

García, M.G., López, C.B., Molina, E.C., Casas, E.E., \& Morales, Y.A.R. (2016). Development and evaluation of the team work skill in university contexts. Are virtual environments effective?. International Journal of Educational Technology in Higher Education, 13(1), 5. doi: https://doi.org/10.1186/s41239-016-0014-1

García Aretio, L. (2017). Educación a distancia y virtual: calidad, disrupción, aprendizajes adaptativo y móvil. RIED. Revista Iberoamericana de Educación a Distancia, 20(2), 925. doi: https://doi.org/10.5944/ried.20.2.18737

Gea, M., Montes, R., Rojas, B., Marin, A., Cañas, A., Blanco, I., \& Gutierrez, C. (2013). Formación abierta sobre modelos de enseñanza masivos: nuevas tendencias hacia el 
aprendizaje social. En IV Congreso Internacional sobre Calidad y Accesibilidad de la Formación Virtual, 17-19.

Godwin-Jones, R. (2011). Emerging technologies: Mobile apps for language learning. Language Learning \& Technology, 15(2), 2-11.

Hanover Reseach (2011). Distance Education Models and Best Practices. Recuperado de https://www.imperial.edu/ivc/files/Distance_Education_Models_and_Best_Practices.pd $\mathrm{f}$

Harrell, I.L. (2008). Increasing the Success of Online Students. Inquiry, 13(1), 36-44.

Hootsuite. (2017). We are social. Recuperado de https://wearesocial.com/special-reports/digitalin-2017-global-overview

Hung, J. L., \& Zhang, K. (2012). Examining mobile learning trends 2003-2008: A categorical meta-trend analysis using text mining techniques. Journal of Computing in Higher education, 24(1), 1-17. doi: https://doi.org/10.1007/s12528-011-9044-9

Hurd, S., Beaven, T., y Ortega, A. (2001). Develop3ing autonomy in a distance language learning context: issues and dilemmas for course writers. System, 29(3), 341-355. Doi: https://doi.org/10.1016/S0346-251X(01)00024-0

Jackson, N.B. (2013). On MOOCs and some possible futures for higher education. Recuperado de http://noelbjackson.wordpress.com/2013/06/01/on-moocs-and-some-possiblefutures-for-higher-ed

Jansen, D., Rosewell, J., y Kear, K. (2017). Quality Frameworks for MOOCs. In Open Education: from OERs to MOOCs (pp. 261-281). Heidelberg: Springer. doi: https://doi.org/10.1007/978-3-662-52925-6_14

Jordano, M., Bárcena, E., y Talaván, N. (2013) Metacognitive and attitudinal issues in second language collaborative projects: Improving individual self-awareness and collective dynamics.

Keegan, D. (1996). Foundations of distance education. Psychology Press.

King, A. (1993). From sage on the stage to guide on the side. College teaching, 41(1), 30-35. doi: https://doi.org/10.1080/87567555.1993.9926781

Knight, P. (2003). The Development of EFL Methodology. En C. Candlin y N. Mercer (Ed.), English Language Teaching in its Social Context. Londres: Routledge, 160-173.

Kong, S. C., Looi, C. K., Chan, T. W., \& Huang, R. (2017). Teacher development in Singapore, Hong Kong, Taiwan, and Beijing for e-Learning in school education. Journal of Computers in Education, 4(1), 5-25. doi: https://doi.org/10.1007/s40692-016-0062-5

Kukulska-Hulme, A., Traxler, J., \& Pettit, J. (2007). Designed and user-generated activity in the mobile age. Journal of Learning Design, 2(1), 52-65. doi: https://doi.org/10.5204/jld.v2i1.28

Leach (2012). The Future of Language Learning: Using Technology to Eliminatre the Affective Filter. Worcester Polytechnic Institute. Recuperado de http://gamesthatdomore.com/wpcontent/uploads/2012/07/OLeach_AffectiveFilter.pdf

Looi, C. K., Seow, P., Zhang, B., So, H.J., Chen, W., y Wong, L.H. (2010). Leveraging mobile technology for sustainable seamless learning: a research agenda. British Journal of Educational Technology, 41(2), 154-169. doi: https://doi.org/10.1111/j.14678535.2008.00912.x

Macness, J. (2013). cMOOCs and XMOOCs - key differences. Jenny Connected. Recuperado de http://jennymackness.wordpress.com/2013/10/22/cmoocs-and-xmoocs-key-differences

Martín-Monje, E., \& Barcena, E. (Ed.) (2015). Language MOOCs: Providing Learning, Transcending Boundaries. Varsovia: Monton de Gruyter. doi: https://doi.org/10.2478/9783110420067

Martín-Monje, E., \& Talaván, N. (2014). The I-AGENT project: Blended learning proposal for professional English integrating MOODLE with classroom work for the practice of oral skills. En E. Bárcena, T. Read y J. Arús (Ed.), Languages for Specific Purposes in the Digital Era. Heidelberg: Springer, 45-67. doi: https://doi.org/10.1007/978-3-319-022222_3

Martín-Monje, E., Bárcena, E., \& Read, T. (2013). Exploring the affordances of Massive Open Online Courses on second languages. En Proceedings of UNED-ICDE (International 
Council for Open and Distance Education). Madrid: UNED. Recuperado de http://unedicde2013.uned.es/?lang=es

McGhee, R., \& Kozma, R. (2007). New teacher and student roles in the technology-supported classroom. Annual Meeting of the American Educational Research Association. Seattle, WA. Recuperado de http://www.aera.net/Events-Meetings/Annual-Meeting/PreviousAnnual-Meetings/2007-Annual-Meeting

Moore, M. G., \& Kearsley, G. (2011). Distance education: A systems view of online learning. Boston, MA: Cengage Learning.

Morrison, D. (2013). The Ultimate Student Guide to xMOOCs and cMOOCs. Recuperado de http://moocnewsandreviews.com/ultimate-guide-to-xmoocs-and-cmoocso

National Capital Language Resource Center (2004). The post-secondary education learning strategies resource guide. Washington, DC: National Capital Language Resource Center.

Oppenheimer, T. (1997). The Computer Delusion. Atlantic Monthly, 1, 45-62.

Pappano, L. (2012). The Year of the MOOC. The New York Times. Recuperado de http://www.nytimes.com/2012/11/04/education/edlife/massive-open-online-courses-aremultiplying-at-a-rapid-pace.html?pagewanted=all\&_r=0

Pérez Pérez, N., Bernabeu Pérez, I., González Gómez, C., Girón Méndez, S., Navarro Soria, I., \& Heliz Llopis, J. (2012). Metodologías docentes y nuevas tecnologías para la coordinación y la práctica docente. En J.D. Álvarez Teruel, M.T. Tortosa Ibález y N. Pellín Buades (Ed.) Diseño de Acciones de Investigación en Docencia Universitaria, 1956-1974.

Piaget, J. (1947). The Psychology of Intelligence (Londres: Routledge, 2001). doi: https://doi.org/10.4324/9780203278895

Prensky, M. (2009). From Digital Inmigrants and Digital Natives to Digital Wisdom. Innovate online, 5(3). Recuperado de http://www.innovateonline.info/pdf/vol5_issue3/H._Sapiens_Digital_From_Digital_I mmigrants_and_Digital_Natives_to_Digital_Wisdom.pdf

Read, T. (2014). The architecture of Language MOOCs. En E. Martín-Monje y E. Bárcena (Ed.), 91-105.

Read, T., \& Barcena, E. (2015). Toward Mobile Assisted Language MOOCs. En A. Mesquita y P. Peres (Ed.) Furthering Higher Education Possibilities Through Massive Open Online Courses. Hershey, PE: IGI Global. doi: https://doi.org/10.4018/978-1-4666-82795.ch011

Read, T., Barcena, E., Traxler, J., \& Kukulska-Hulme, A. (2017). Toward a Mobile Open and Social Language Learning Paradigm.

Read, T., Barcena, E., \& Kukulska-Hulme, A. (2015). Mobile and Massive Language Learning. En E. Martin-Monje, I. Elorza y B. Garcia-Riaza (Ed.) Technology-Enhanced Language Learning for Specialized Domains: Practical applications and mobility. Londres: Routledge.

Read, T., \& Bárcena, E. (2014). Modelling User Linguistic Communicative Competences for Individual and Collaborative Learning. En J. Wyatt, D. Petters y D. Hogg (Ed.) From Animals to Robots and Back: Reflections on Hard Problems in the Study of Cognition, Berlín: Springer, 205-218. doi: https://doi.org/10.1007/978-3-319-06614-1_14

Read, T., Bárcena, E., \& Rodrigo, C. (2010). Modelling ubiquity for second language learning. International Journal of Mobile Learning and Organisation, 4(2), 130-149. doi: https://doi.org/10.1504/IJMLO.2010.032633

Reed, M.S., Evely, A. C., Cundill, G., Fazey, I., Glass, J., Laing, A. Newig, J. Parrish, B. Prell, C. Raymond, C., \& Stringer, L.C. (2010). What is Social Learning?. Ecology and Society 15(4). doi: https://doi.org/10.5751/ES-03564-1504r01

Romeo, K. (2012). Language Learning MOOCs? Hive Talkin. Recuperado de https://www.stanford.edu/group/ats/cgi-bin/hivetalkin/?p=3011

Sestini, F. (2012). Collective awareness platforms: engines for sustainability and ethics. IEEE Technology and Society Magazine, 31(4), 54-62. doi: https://doi.org/10.1109/MTS.2012.2225457

Sharples, M. (2000). The design of personal mobile technologies for lifelong learning. Computers \& Education, 34(3), 177-193. doi. https://doi.org/10.1016/S0360-1315(99)00044-5 
Siemens, G. (2005). Connectivism: Learning as network-creation. ASTD Learning News, 10(1), $1-28$.

Thorne, S., \& Payne, J.S. (2005). Evolutionary Trajectories, Internet- mediated Expression, and Language Education. CALICO Journal, 22(3), 371-397. doi: https://doi.org/10.1558/cj.v22i3.371-397

Turoff, M. (1991). Computer-Mediated Communication Requirements for Group Support. Journal of Organizational Computing, 1, 85-113. doi: https://doi.org/10.1080/10919399109540151

Vorobel, O., \& Kim, D. (2012). Language teaching at a distance: An overview of research. CALICO Journal, 29(3), 548. doi: https://doi.org/10.11139/cj.29.3.548-562

Vygotsky, L.S. (1978). Mind in society: The development of higher mental process. Cambridge, MA: Harvard University Press.

Wang, Y., \& Sun, C. (2001). Internet-based real time language education: Towards a fourth generation distance education. CALICO Journal, 539-561. doi: https://doi.org/10.1558/cj.v18i3.539-561

Warschauer, M., \& Kern, R. (2000). Network-based language teaching: Concepts and practice. Cambridge: Cambridge University Press. doi: https://doi.org/10.1017/CBO9781139524735

Watters, A. (2012). Top Ed-Tech Trends of 2012: MOOCs. Recuperado de http://hackeducation.com/2012/12/03/top-ed-tech-trends-of-2012-moocs

White, C. (2006). Distance learning of foreign languages. Language Teaching, 39, 247-264. doi: https://doi.org/10.1017/S0261444806003727

Wijayanti, R.H. (2017). Heuristic Reflection for Teachers for Better Learning Teaching Interaction. Indonesian Journal of English Language Studies (IJELS), 1(1), 113.

Wong, L.H. (2012). A learner-centric view of mobile seamless learning. British Journal of Educational Technology, 43(1), 19-23. doi: https://doi.org/10.1111/j.14678535.2011.01245.x

Wong, L.H., y Looi, C.K. (2011). What seams do we remove in mobile-assisted seamless learning? A critical review of the literature. Computers \& Education, 57(4). doi: https://doi.org/10.1016/j.compedu.2011.06.007

Wong, L. H., Chai, C. S., Chin, C. K., Hsieh, Y. F., y Liu, M. (2011). Leveraging Ubiquitous Technology for Seamless Language Learning: From "Move, Idioms!" to MyCLOUD. Proceedings of World Conference on Mobile and Contextual Learning, 231-239.

Yeager, C., Hurley-Dasgupta, B., y Bliss, C. A. (2013). CMOOCs and global learning: an authentic alternative. Journal of Asynchronous Learning Networks, 17(2). https://doi.org/10.24059/olj.v17i2.347 